\title{
Analytical Model of the Slotless Double-Sided Axial Flux Permanent- Magnet Brushless Machines
}

\author{
A. Ghaffari \\ Department of Electrical and Electronics Engineering, Shiraz University of Technology, Shiraz, I.R. Iran \\ a.ghaffari@sutech.ac.ir
}

Received: 20 March 2020; Accepted: 26 April 2020; Available online: 5 June 2020

\begin{abstract}
Analytical approaches, if possible, are suggested for saving the simulation time in the design stage of the electrical machines. This benefit is highlighted when the optimization issues including too many iterations are desired. Hence, this paper presents a 2-D analytical model for magnetic field distribution based on the sub-domain method in a slotless double-sided axial flux permanent-magnet (PM) brushless machines (AFPMBMs) with internal-rotor-external-stators. According to this method, the machine cross-section is divided into the appropriate number of sub-regions and the related partial differential equations (PDEs) extracted from Maxwell equations are formed for magnetic vector potential in each sub-region. Applying curl on the obtained results leads to calculating the magnetic flux density components in each sub-region. Based on the superposition theorem, the analytical procedure is utilized in the two separate steps where in the first step the magnetic flux is originated by only PMs with various magnetization patterns (i.e., parallel, ideal Halbach, 2-segment Halbach and bar magnet in shifting magnetization patterns) and the armature currents are zero. In the second step, all PMs are inactivated and only armature currents affect the magnetic flux distribution. Finally, the obtained analytical results are compared with those of the Finite element method (FEM) to confirm the accuracy of the proposed analytical model. The extracted results reveal the benefit of the analytical model for replacing instead of the FEM to predict the magnetic flux density component in the presented AFPMBMs in a shorter time.
\end{abstract}

Keywords: Analytical design; Axial flux motor; Maxwell equations; Permanent-magnet; Sub-domain technique.

\section{Introduction}

At the moment, electric machines’ presence and their vast kinds of applications in industries are undeniable. Among them, axial flux permanent magnet machines (AFPMs) are more preferred on compactable machines with high torque/weight ratio. From the perspective of structure, we can divide them into single-sided, double-sided and multi-stages [1]. The double-sided structure includes external-rotor-internal- stator (TORUS) and internalrotor-external-stator (AFIR) in terms of rotor and stator positions and each one has its own advantages. Extensive researches have been carried out over these structures that can be classified as [2]:

1) Analytical or numerical models (0-D, 1-D, 2-D, 3-D).

2) PMs configurations such as surface-mounted, surface-inset, buried PMs, spoke PMs, etc.;

3) Magnetization patterns such as parallel, ideal Halbach, 2-segment Halbach, bar magnets in shifting directions, patterns, etc.

4) Magnetic fields quotations formulated in Cartesian, cylindrical or polar coordinates;

5) Machines' structure such as slotted, slotless, coreless, etc.

6) PM shapes such as rectangular, trapezoidal or circular.

7) Considering or not considering the saturation effect.

8) Magnetic field calculations based on PMs, the armature reaction or both of them.

Analytical and numerical models were developed for analyzing the electrical machines. For instance, In [3], the authors tried to analyze the 3-D magnetic field distribution by using the Fast Fourier Transform (FFT) where the 3-D analytical model including more complexity compared with other analytical models. Authors in [4] mentioned that in the finite element method, a large number of air areas considered surround the conductors in order to satisfy the boundary conditions at infinity. They developed a hybrid finite element/boundary element (FE/BE) method to avoid such areas aiming to decrease magnetic field calculation. In [5] calculation of 3-D magnetic fields by using integral transformation has been developed. The scalar magnetic potential is obtainable considering the discrete Fourier transformation and the Hankel transformation over angle coordinate and radial coordinate, respectively. In [6], a 3-D magnetic field solution describes an enhanced three dimensional (3-D) field reconstruction method for modeling an axial flux permanent magnet machine. 
As mentioned, magnetic field computation in an electric machine may be achieved using the analytical and numerical models [7-55]. For instance, the analytical models were investigated in [8-19, 21-27, 29, 32-35, 37, 40, 44-48, 50-53] and the numerical ones were explained in [7], [36],[39], [41],[42], [49]. The main drawback of the numerical method is related to their high computational burden and more simulation time. So, some novel methods such as image method [20], [38] and field reconstruction method [28], [30]-[31], [49] aiming to reduce the amount of calculation of FEM method, have been developed in the previous studies. However in [20], the authors claim that their results are more realistic than the quasi-3-D method, but no comparison is reported. In quasi-3-D method [11],[12], [14], [16], [29], [37], [40] it is commonly assumed that the machine is composed of several linear machines. Several cutting planes are chosen and analyzed from the machine and the total results of the machine are obtained by adding the extracted results of each plane. In [11] and [12] the analytical methods are studied for TORUS-type, in cylindrical coordinate and for single-sided structure with Halbach and axially magnets arrangement, in polar coordinate, respectively that the benefits of the analytical model for saving time compared with the numerical ones were described. In [14] and [16] authors investigated analytical methods for single-sided slotted stator structure in cylindrical coordinate. In [29], Parviainen et al. obtained flux density distribution in the air gap region considering stator slot openings. The analytical method is studied for a slotted stator AFIR-type PM machine. Alipour et al. [37], employed Schwartz-Christoffel transformation in order to calculate circumferential and perpendicular components of the air gap flux density due to the PMs and the armature current. Tiegna et al. [40], considered and extended a function that reveals radial dependence of the magnetic field and it is applicable for any type of PMs. It is been framed by composing FEM and multi-slice AM methods which are able to consider the end effects of the machine. 2-D analytical method also investigated in [8], [10], [15], [17], [19], [21], [24], [26], [33-35], [44-48], [50], [53]. In [8], the authors investigated a 2-D analytical method for magnetic field calculations in PMs and air gap regions for a single-sided structure in cylindrical coordinate [10], Cartesian coordinate [54] and for a double-sided slotted stator in [15]. The 2-D analytical method also was described for slotted stator TORUS-type [19, 24], for calculation of cogging torque and EMF [21]. Also, this analytical model is employed for calculating torque, EMF and inductances for a slotted stator with surface-inset PMs [26]. Besides, the 2-D analytical method also investigated different kinds of machines such as radial flux [33], linear machine [35], flux switching machine with double-sided structure [43] and single-sided structure with two flux return plates [44]. Zhu et al. [34], studied an accurate sub-domain model for a slotted stator structure with radial and parallel magnetization patterns in polar coordinate.

The considered papers reveal that the analytical models, if possible, are preferred due to the following three reasons:

1) The analytical models are faster than the numerical ones which are essential for the optimization issues with numerous iterations.

2) The analytical method provides a better understanding of the system. It helps to comprehend governing equations in the electrical machines.

3) The analytical model is more flexible for modifying motor specifications, such as the dimensions of motor or the number of PMs, in spite of the numerical methods which changing the specifications requires remodeling the machine.

It is necessary to mention that, some authors of the analytical models assumed infinite permeability for cores to simplify the analytical model that leads to having no sense about the magnetic flux density distribution in the cores [7], [22], [33]. Also, some authors investigated just PM effects on the magnetic field distribution and the effect of armature currents was not considered [49],[52], or the authors analyzed the effect of armature currents and the PM effects were not described [35], [55]. Moreover, there is no explanation of various magnetization patterns in most of the previous researches. Therefore, finding an accurate analytical model for the electrical machine with finite permeability of cores is essential to predict the magnetic flux density due to PMs with various magnetization patterns and armature currents.

The main contribution of this paper is related to defining an analytical model for the slotless AFPMBM under the study. In this study, the permeability of cores is assumed to be finite and both armature currents and PMs effect including various magnetization patterns, such as parallel, ideal Halbach, 2-segment Halbach and bar magnet in shifting magnetization patterns, are described to obtain the magnetic flux density components in each sub-region by employing the sub-domain technique and applying Maxwell equations.

\section{Methodology}

The electromagnetic problem initiates by invoking a set of assumptions to enable the analytical solution of the governing partial differential equations (PDEs) originating from Maxwell's equations. Magnetization patterns are expressed in terms of their Fourier series expansion.

In this paper, the formulation is based on the magnetic vector potential, which leads to a set of Laplace and Poisson equations. Based on the governing equations and a set of boundary conditions, a general solution is 
assigned to each region [7]. The geometry of the presented slotless AFPMBM under the study, which consists of eleven sub-regions, is shown in Fig. 1 . Noted that $r, \theta$ and $z$ in polar coordinate are replaced respectively with $z, x$ and $y$ in Cartesian coordinate to model the motor under the study in the Cartesian coordinate. Also, this assumption leads to replacing the redial magnetization pattern with the parallel one.

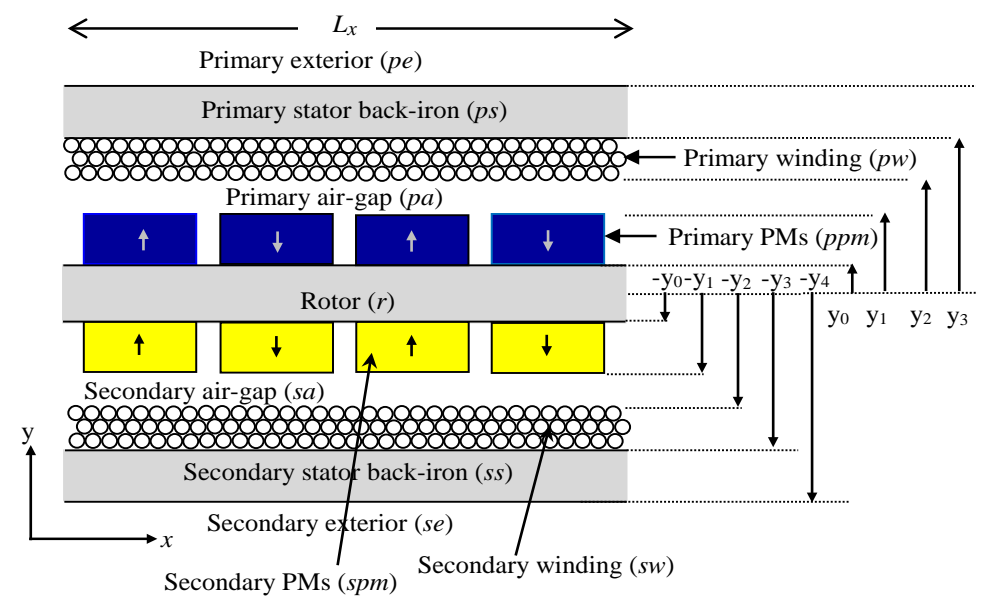

Fig. 1. Cross-section and sub-regions of the slotless AFPMBM under the study

\subsection{List of assumptions}

The following assumptions are made for defining the proposed analytical model:

1) End effects are ignored.

2) In the case of $2 D$ problems in Cartesian coordinates, magnetic vector potential, $\boldsymbol{A}$, magnetization pattern, $\boldsymbol{M}$, magnetic flux density vector, $\boldsymbol{B}$, and current density vector, $\boldsymbol{J}$, are described as follow: $\boldsymbol{A}=\left[0,0, A_{z}(x, y)\right] ; \boldsymbol{M}=$ $\left[M_{x}(x), M_{y}(x), 0\right] ; \boldsymbol{B}=\left[B_{x}(x, y), B_{y}(x, y), 0\right] ; \boldsymbol{J}=\left[0,0, J_{z}(x, t)\right]$.

3) All materials are isotropic.

4) The media have finite relative permeability.

5) The saturation effects are neglected.

6) The motor has a slotless stator structure.

7) Eddy current reaction field is neglected.

\subsection{Governing PDEs}

The related PDEs in all sub-region of the presented slotless AFPMBM can be defined as follow:

$$
-\nabla^{2} \boldsymbol{A}=\mu_{0} \mu_{r} \boldsymbol{J}+\mu_{0} \nabla \times \boldsymbol{M}
$$

The above equation is employed into 2 separate steps for calculating the magnetic flux density vector in each sub-region. In the first step, the magnetic flux density is obtained by only PMs and armature currents are zero and in the second step, the magnetic flux density is originated due to only armature reaction and all PMs are inactivated.

\subsubsection{Magnetic flux density due to only PMs}

In this step, only PMs affect the magnetic flux density distribution in all sub-regions that leads to extracting two categories of the PDEs. The first group pertinent to all sub-regions except for PMs and the related PDEs in these sub-regions are Laplace equations that are defined as follows:

$$
\nabla^{2} A^{i}=0 \quad i=\{p e, p s, p w, p a, r, s a, s w, s s, s e\}
$$

Solving the following PDEs leads to predict the magnetic vector potential in each sub-region of this group as follows:

$$
A_{z}^{i}(x, y)=\sum_{n=1}^{\infty}\left(a_{n}^{i} \sinh \left(\alpha_{n} y\right)+b_{n}^{i} \cosh \left(\alpha_{n} y\right)\right) \cos \left(\alpha_{n} x\right)+\left(c_{n}^{i} \sinh \left(\alpha_{n} y\right)+d_{n}^{i} \cosh \left(\alpha_{n} y\right)\right) \sin \left(\alpha_{n} x\right)
$$

where $\alpha_{n}=n \pi / \tau_{p}$ in which $\tau_{p}$ is pole pitch. 
The second group of equations is related to PMs sub-regions that the relevant equations are Poisson ones described as follows:

$$
\nabla^{2} \boldsymbol{A}^{P M}=-\mu_{0} \nabla \times \boldsymbol{M}
$$

where $\mu_{0}$ is free space permeability. Noted that in the sub-domain technique the magnetization patterns are defined based on their Fourier series expansions to calculate the tangential components, $M_{x}$, and normal components, $M_{y}$, as follow:

$$
\begin{aligned}
& \boldsymbol{M}=M_{x} a_{x}+M_{y} a_{y} \\
& M_{x}(x)=\sum_{n=1}^{\infty} m_{x n} \cos \left(\alpha_{n} x\right) \\
& M_{y}(x)=\sum_{n=1}^{\infty} m_{y n} \sin \left(\alpha_{n} x\right)
\end{aligned}
$$

where $m_{x n}$ and $m_{y n}$ are Fourier series components. The amplitude of these components for the investigated magnetization patterns and their illustrative representation are described in Table 1 and Fig. 2, respectively. According to the defined Maxwell equations in PMs sub-region and the magnetization pattern components, the following equation can be extracted for PMs sub-regions.

$$
\begin{aligned}
& A_{z}^{P M}(x, y)=\sum_{n=1}^{\infty}\left(a_{n}^{P M} \sinh \left(\alpha_{n} y\right)+b_{n}^{P M} \cosh \left(\alpha_{n} y\right)+\frac{\mu_{0}}{\alpha_{n}} m_{x n}\right) \cos \left(\alpha_{n} x\right)+\left(c_{n}^{P M} \sinh \left(\alpha_{n} y\right)+\right. \\
& \left.d_{n}^{P M} \cosh \left(\alpha_{n} y\right)-\frac{\mu_{0}}{\alpha_{n}} m_{y n}\right) \sin \left(\alpha_{n} x\right)
\end{aligned}
$$

Applying curl on the determined magnetic vector potential in each sub-region leads to obtaining the magnetic flux density as follows:

$$
\begin{aligned}
& B_{x}^{i}(x, y)=\sum_{n=1}^{\infty} \alpha_{n}\left[\left(a_{n}^{i} \cosh \left(\alpha_{n} y\right)+b_{n}^{i} \sinh \left(\alpha_{n} y\right)\right) \cos \left(\alpha_{n} x\right)+\left(c_{n}^{i} \cosh \left(\alpha_{n} y\right)+\right.\right. \\
& \left.\left.d_{n}^{i} \sinh \left(\alpha_{n} y\right)\right) \sin \left(\alpha_{n} x\right)\right] \\
& \quad B_{y}^{i}(x, y)=\sum_{n=1}^{\infty} \alpha_{n}\left[\left(a_{n}^{i} \sinh \left(\alpha_{n} y\right)+b_{n}^{i} \cosh \left(\alpha_{n} y\right)\right) \sin \left(\alpha_{n} x\right)-\left(c_{n}^{i} \sinh \left(\alpha_{n} y\right)+\right.\right. \\
& \left.\left.d_{n}^{i} \cosh \left(\alpha_{n} y\right)\right) \cos \left(\alpha_{n} x\right)\right] \\
& B_{x}^{P M}(x, y)=\sum_{n=1}^{\infty} \alpha_{n}\left[\left(a_{n}^{P M} \cosh \left(\alpha_{n} y\right)+b_{n}^{P M} \sinh \left(\alpha_{n} y\right)\right) \cos \left(\alpha_{n} x\right)+\left(c_{n}^{P M} \cosh \left(\alpha_{n} y\right)+\right.\right. \\
& \left.\left.d_{n}^{P M} \sinh \left(\alpha_{n} y\right)\right) \sin \left(\alpha_{n} x\right)\right] \\
& B_{y}^{P M}(x, y)=\sum_{n=1}^{\infty} \alpha_{n}\left[\left(a_{n}^{P M} \sinh \left(\alpha_{n} y\right)+b_{n}^{P M} \cosh \left(\alpha_{n} y\right)+\frac{\mu_{0}}{\alpha_{n}} m_{x n}\right) \sin \left(\alpha_{n} x\right)-\left(c_{n}^{P M} \sinh \left(\alpha_{n} y\right)+\right.\right. \\
& \left.\left.d_{n}^{P M} \cosh \left(\alpha_{n} y\right)-\frac{\mu_{0}}{\alpha_{n}} m_{y n}\right) \cos \left(\alpha_{n} x\right)\right]
\end{aligned}
$$

\begin{tabular}{|c|c|c|}
\hline Magnetization pattern & $m_{x n}$ & $m_{y n}$ \\
\hline Parallel & 0 & $M_{1 n}$ \\
\hline Ideal halbach & $M$ & $-M$ \\
\hline 2-Segments & $-\frac{4 M}{n \pi} \sin \left(n \pi \frac{k_{x}}{2}\right)$ & $M_{2 n}$ \\
\hline Bar magnets in shifting directions & $M_{3 n}$ & $M_{4 n}$ \\
\hline \multicolumn{3}{|c|}{$\begin{array}{l}* M=\frac{B_{r e m}}{\mu_{0}} \text { where } B_{\text {rem }} \text { is PM Remanence flux density, } M_{1 n}=\frac{4 M}{n \pi}\left[\sin \left(\frac{n \pi}{2}\right) \sin \left(\frac{\alpha_{n} \tau_{m}}{2}\right)\right] \text { where } \tau_{m} \text { is each PM width. } M_{2 n}= \\
\frac{-2 M}{n \pi}\left[\cos \left(\mathrm{n} \pi\left(\frac{k_{x}}{2}+k_{y}\right)-\cos \left(\mathrm{n} \pi\left(\frac{k_{x}}{2}\right)\right], M_{3 n}=4 n M \frac{\alpha_{p}^{2}}{\pi} \sin \left(\frac{n \pi}{2}\right) \cos \left(n \pi \frac{\alpha_{p}}{2}\right) /\left(n \alpha_{p}\right)^{2}-1, M_{4 n}=-4 M \frac{\alpha_{p}}{\pi} \sin \left(\frac{n \pi}{2}\right) \cos \left(n \pi \frac{\alpha_{p}}{2}\right) /\right.\right. \\
\left(n \alpha_{p}\right)^{2}-1, \alpha_{p}=\frac{\tau_{m}}{\tau_{p}} \cdot k_{x} \text { and } k_{y} \text { are respectively } x \text { and } y \text {-direction magnetized PM width to the pole pitch ratio for 2-segment } \\
\text { Halbach. }\end{array}$} \\
\hline
\end{tabular}

For considering the rotor rotation $x$ must be substituted by $x-d$ which $d$ is the rotor motion and described as:

$$
d=v t+d_{0}
$$

where $v$ is converted rotor angular velocity $(\omega)$ to the linear translation speed, $t$ is time and $d_{0}$ is initial rotor position.

Table 1. The normal and tangential components of the magnetization patterns. 
Parallel magnetization pattern

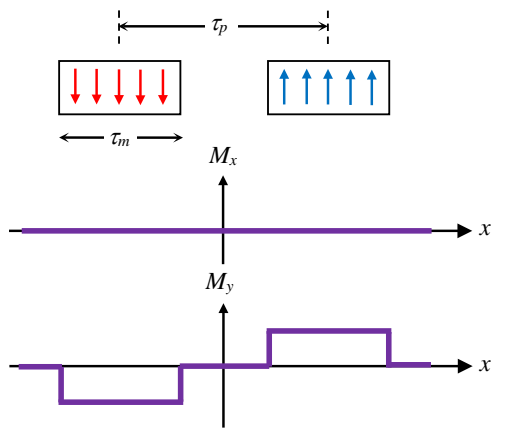

Ideal Halbach magnetization pattern
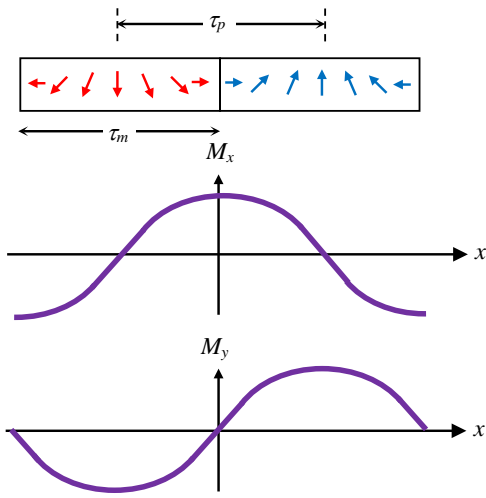

2-Segment Halbach magnetization pattern

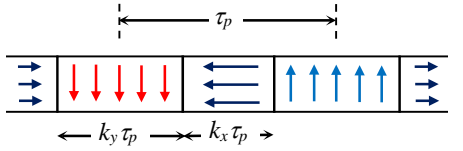

(a)

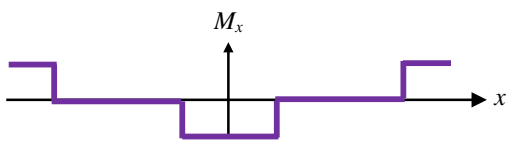

(b)

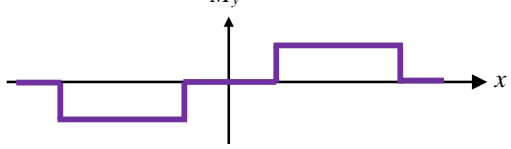

Bar magnet in shifting direction magnetization pattern
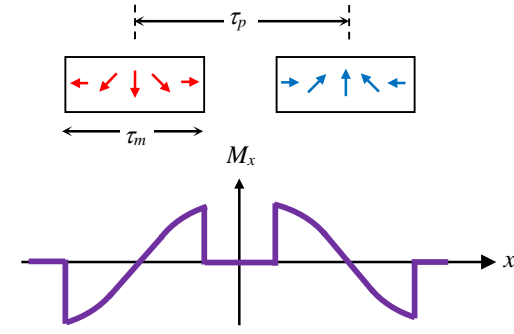

(b)

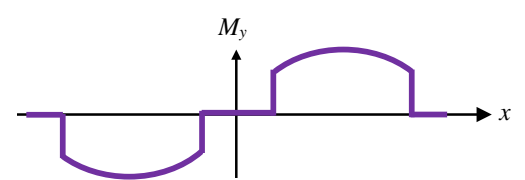

Fig. 2. (a) Illustrative representation of each magnetization pattern; (b) tangential; and (c) normal components.

\subsubsection{Magnetic flux density due to only armature currents}

In this step, PMs should be inactive to estimate the effects of armature currents on the magnetic flux density distribution. Two categories for the PDEs Like the previous step are formed in this step. The first group includes all sub-regions except for windings sub-regions where the related PDEs for this group is Laplace one that is described as follows:

$$
\nabla^{2} A^{i}=0 \quad i=\{p e, p s, p a, p p m, r, s p m, s a, s s, s e\}
$$

Solving the above equations leads to obtaining similar magnetic flux density expression to Eqs. (9)- (10).

The second group of this step comprises the windings that the explained PDEs in this group is Poisson one as follows:

$$
\nabla^{2} \boldsymbol{A}^{w}=-\mu_{0} \boldsymbol{J} \quad w=\{p w, s w\}
$$

The Fourier series expansion of armature currents density is necessary to solve the above equation. For this aim, armature current density is expressed as follow:

$$
J(x, t)=\sum_{n=1}^{\infty} J_{1 n} \sin \left(\alpha_{n} x\right)+J_{2 n} \cos \left(\alpha_{n} x\right)
$$

where $J_{1 n}$ and $J_{2 n}$ are Furrier series components of current density. These components for three phases motor are calculated as follow:

$$
\begin{aligned}
& J_{1 n}=-\frac{2 N_{t} \tau_{p}}{3\left(y_{3}-y_{2}\right)} \frac{\cos \left(\frac{2 n \pi}{3}\right)-\cos \left(\frac{n \pi}{3}\right)}{n \pi} \times\left(i_{a}+i_{b} \cos \left(\frac{2 n \pi}{3}\right)+i_{c} \cos \left(\frac{2 n \pi}{3}\right)\right) \\
& J_{2 n}=-\frac{2 N_{t} \tau_{p}}{3\left(y_{3}-y_{2}\right)} \frac{\cos \left(\frac{2 n \pi}{3}\right)-\cos \left(\frac{n \pi}{3}\right)}{n \pi} \times\left(-i_{b} \sin \left(\frac{2 n \pi}{3}\right)+i_{c} \sin \left(\frac{2 n \pi}{3}\right)\right) .
\end{aligned}
$$


where $N_{t}$ is the number of turns for each coil where excited by the following currents:

$$
\begin{aligned}
& i_{a}(t)=I_{m} \sin (\omega t) \\
& i_{b}(t)=I_{m} \sin \left(\omega t-\frac{2 \pi}{3}\right) \\
& i_{c}(t)=I_{m} \sin \left(\omega t+\frac{2 \pi}{3}\right)
\end{aligned}
$$

where $I_{m}$ is the maximum phase current. Therefore, the related solution of the magnetic vector potential in the windings sub-regions is determined as follows:

$$
\begin{aligned}
& A_{z}^{w}(x, y)=\sum_{n=1}^{\infty}\left(a_{n}^{w} \sinh \left(\alpha_{n} y\right)+b_{n}^{w} \cosh \left(\alpha_{n} y\right)+\frac{\mu_{0}}{\alpha_{n}^{2}} J_{2 n}\right) \cos \left(\alpha_{n} x\right)+\left(c_{n}^{w} \sinh \left(\alpha_{n} y\right)+\right. \\
& \left.d_{n}^{w} \cosh \left(\alpha_{n} y\right)+\frac{\mu_{0}}{\alpha_{n}^{2}} J_{1 n}\right) \sin \left(\alpha_{n} x\right)
\end{aligned}
$$

Similar to the previous section, applying curl on the obtained magnetic vector potential leads to calculating the magnetic flux density components.

\subsection{The boundary conditions}

The calculated magnetic vector potential extracted from Maxwell equations in each sub-region includes 4 variables. Therefore, 44 variables are available and it is necessary to form 44 equation to predict the magnetic vector potential in the slotless AFPMBM under the study. Noted that, according to the geometry structure and applied coordinate system, some coefficients (i.e. $a_{n}^{p e}, c_{n}^{p e}, b_{n}^{s e}, d_{n}^{s e}$ ) must be zero and 40 variables are extracted based on the defined PDEs in each sub-region. These 40 variables are:

$$
\begin{aligned}
& \quad b_{n}^{p e}, d_{n}^{p e}, a_{n}^{p s}, b_{n}^{p s}, c_{n}^{p s}, d_{n}^{p s}, a_{n}^{p w}, b_{n}^{p w}, c_{n}^{p w}, d_{n}^{p w}, a_{n}^{p a}, b_{n}^{p a}, c_{n}^{p a}, d_{n}^{p a}, a_{n}^{p p m}, b_{n}^{p p m}, c_{n}^{p p m}, d_{n}^{p p m}, a_{n}^{r}, b_{n}^{r}, c_{n}^{r}, d_{n}^{r}, \\
& a_{n}^{s p m}, b_{n}^{s p m}, c_{n}^{s p m}, d_{n}^{s p m}, a_{n}^{s a}, b_{n}^{s a}, c_{n}^{s a}, d_{n}^{s a}, a_{n}^{s w}, b_{n}^{s w}, c_{n}^{s w}, d_{n}^{s w}, a_{n}^{s s}, b_{n}^{s s}, c_{n}^{s s}, d_{n}^{s s}, a_{n}^{s e}, c_{n}^{s e} .
\end{aligned}
$$

For calculating these variables the magnetic boundary conditions are employed. These boundary conditions are extracted based on the following equations for the motor under the study:

$$
\begin{aligned}
& \left.H_{x}^{i}(x, y)\right|_{y=Y}=\left.H_{x}^{i+}(x, y)\right|_{y=Y} \quad\left(i, i^{+}, Y\right)=\left\{\begin{array}{l}
\left(p e, p s, y_{4}\right),\left(p s, p w, y_{3}\right),\left(p w, p a, y_{2}\right),\left(p a, p p m, y_{1}\right),\left(p p m, r, y_{0}\right), \\
\left(s p m, r,-y_{0}\right),\left(s p m, s a,-y_{1}\right),\left(s a, s w,-y_{2}\right),\left(s w, s s,-y_{3}\right),\left(s s, s e,-y_{4}\right)
\end{array}\right\} \\
& \left.B_{y}^{i}(x, y)\right|_{y=Y}=\left.B_{y}^{i+}(x, y)\right|_{y=Y}
\end{aligned}
$$

where $\boldsymbol{H}$ is magnetic field intensity vector. All these extracted 40 boundary conditions are described in Appendix.

\section{Results and discussion}

To validate the derived analytical expressions, the magnetic field distributions in each sub-region of the presented AFPMBM are compared with those of FEM to describe the accuracy of the proposed analytical model. For this aim, the motor including the specifications in Table 2 is employed and the extracted results are developed for both analytical and numerical models. The analytical and numerical results of magnetic flux density distribution due to armature currents and PMs in the motor under the study are respectively represented in Figs 3 and 4 where acceptable accuracy between both analytical and numerical models can be observed.

Noted that the facing PMs on both sides of the presented motor with parallel magnetization patterns play an important role in magnetic flux in the rotor. In this study, facing PMs on both sides are magnetized in a different direction which leads to reducing the tangential components of magnetic flux in the rotor. Also, the extracted results reveal that the magnetic flux passes through the PMs in the Halbach magnetization pattern. Therefore, in the investigated magnetization patterns it is possible to eliminate the rotor and replacing it by the material which is lighter or cheaper.

The slotless stator structure of the studied motor results in increasing the magnetic air-gap and the magnetic flux density in originated due to PMs and armature currents have no significant effects on magnetic flux density distribution. Also, the distributed magnetic flux due to ideal Halbach magnetization patterns includes the minimum total harmonic distortion compared with those of other magnetization patterns.

The simulation time has been mentioned as the main benefit of the proposed analytical model. In this study, a computer with 32-GB RAM and TM i7-7700 Processor was employed and the maximum length of $4 \mathrm{~mm}$ in each mesh was considered for the FEM model. In this condition, the analytical model simulation time was 11 times less 
than the numerical model for the studied motor. It means that applying the analytical model in the design stage of the presented slotless AFPMBM results in saving a considerable amount of time.

Table 2. Main design parameters of the proposed axial flux motor under the study

\begin{tabular}{lll}
\hline Parameters & Symbols & Values \\
\hline Rotor back iron height & $2 y_{0}$ & $12 \mathrm{~mm}$ \\
PM height & $y_{1}-y_{0}$ & $6 \mathrm{~mm}$ \\
Air-gap height & $y_{2}-y_{1}$ & $2 \mathrm{~mm}$ \\
Winding height & $y_{3}-y_{2}$ & $10 \mathrm{~mm}$ \\
Stator back iron height & $y_{4}-y_{3}$ & $10 \mathrm{~mm}$ \\
Stator relative permeability & $\mu_{r}^{s}$ & 1000 \\
Mover relative permeability & $\mu_{r}^{r}$ & 1500 \\
PM relative permeability & $\mu_{r}^{P M}$ & 1.05 \\
PM width for the parallel pattern & $\tau_{m}$ & $40 \mathrm{~mm}$ \\
Pole pitch & $\tau_{p}$ & $50 \mathrm{~mm}$ \\
Number of poles & $p$ & 8 \\
$x$-direction magnetized PM width to the pole pitch ratio for 2-segment Halbach & $k_{x}$ & 0.4 \\
y-direction magnetized PM width to the pole pitch ratio for 2-segment Halbach & $k_{y}$ & 0.6 \\
PM Remanence flux density & $B_{r e m}$ & $1.13 \mathrm{~T}$ \\
Peak armature current & $I_{m}$ & $5 \mathrm{~A}$ \\
Number of turns per coil & $N_{t}$ & 76 \\
Number of coils per phase & $N_{c}$ & 4 \\
Filling factor & $K_{f}$ & 0.6
\end{tabular}
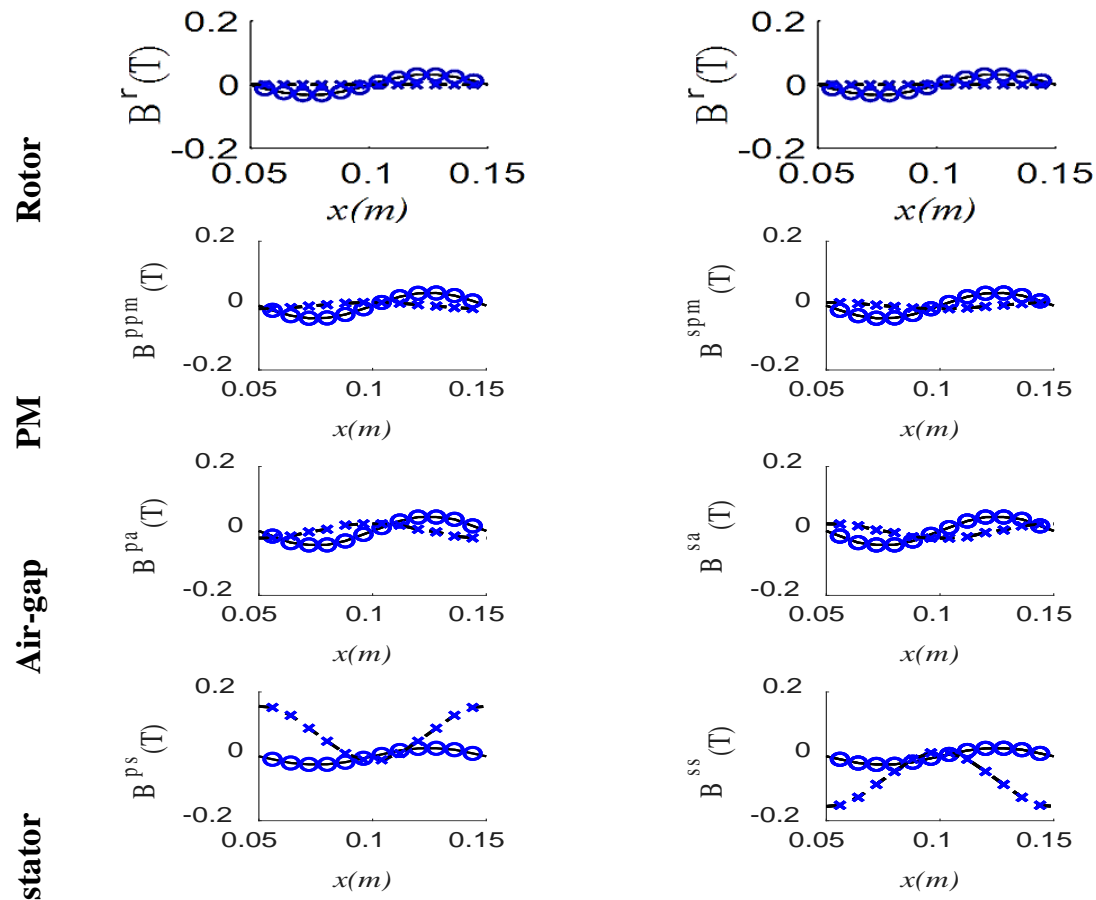

o Numerical results of the normal component of flux density - Analytical results of the normal component of flux density $\times$ Numerical results of the tangential component of flux density - - Analytical results of the tangential component of flux density

Fig. 3. Flux density component in the presented motor due to only armature current 


\section{Parallel}
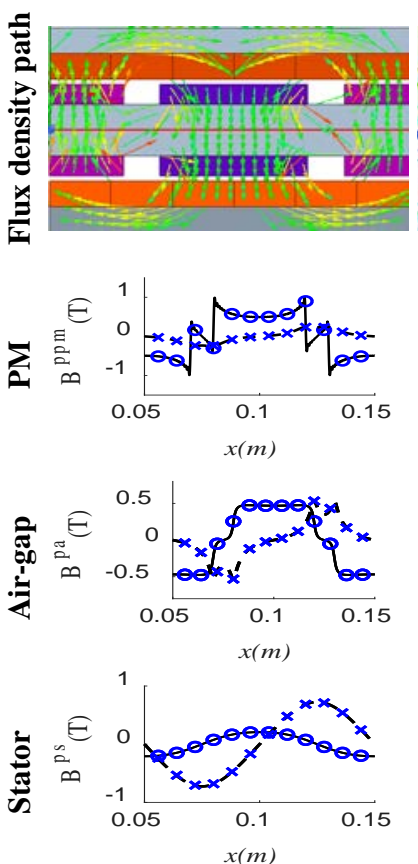

Ideal Halbach
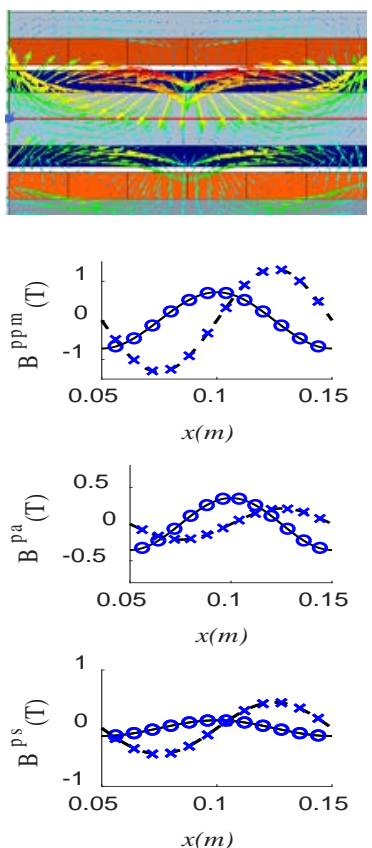

(a): Primary sub-regions of the presented slotless AFPMBM
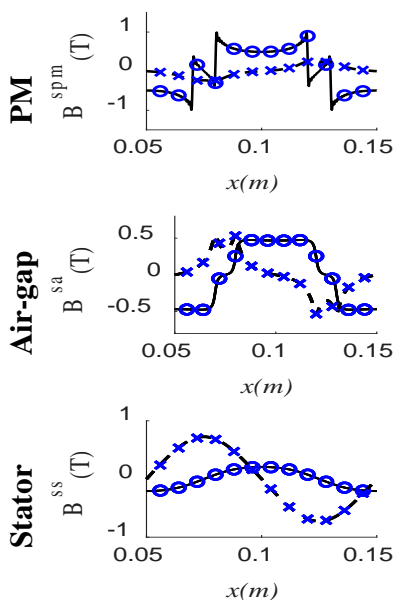

Ideal Halbach
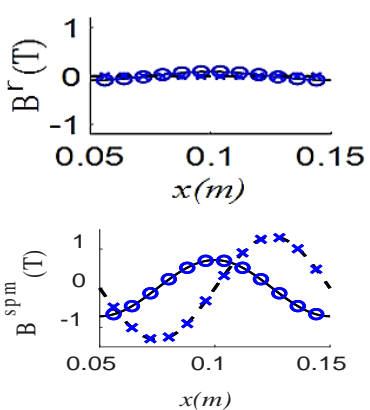

2-segment Halbach
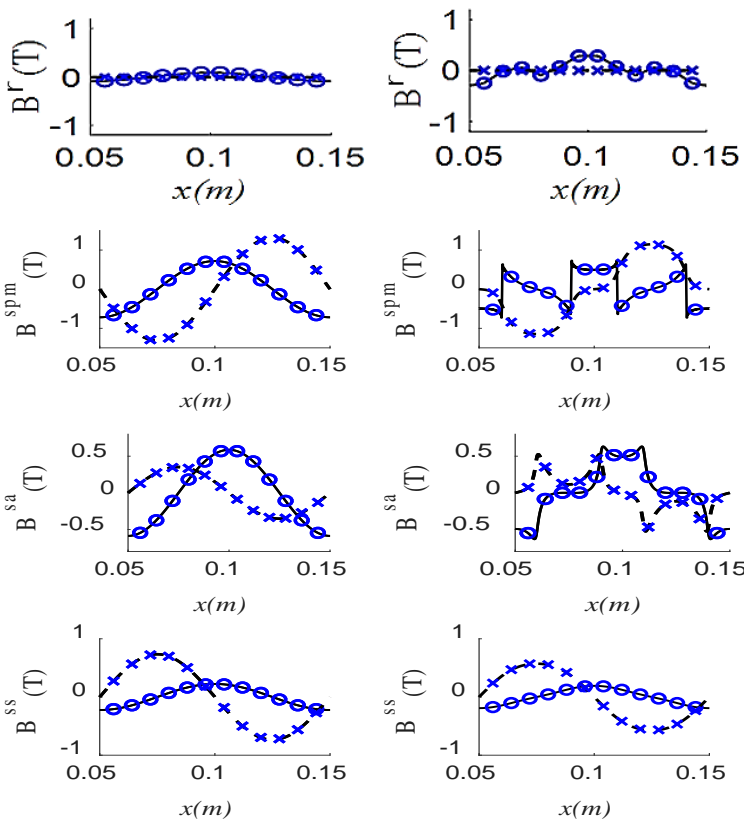

2-segment Halbach
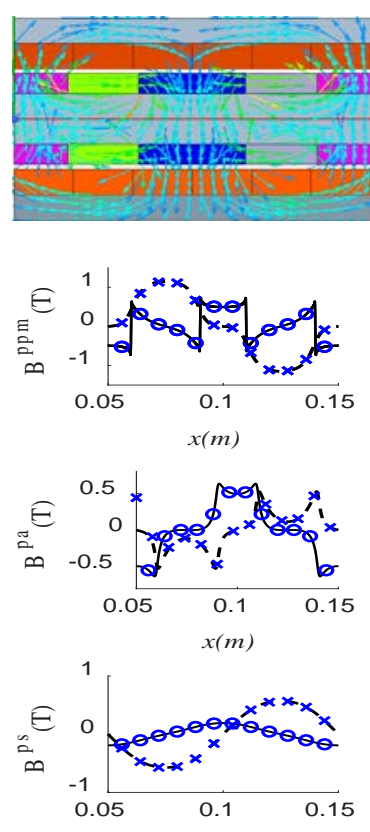

$x(m)$
Bar magnets in shifting directions
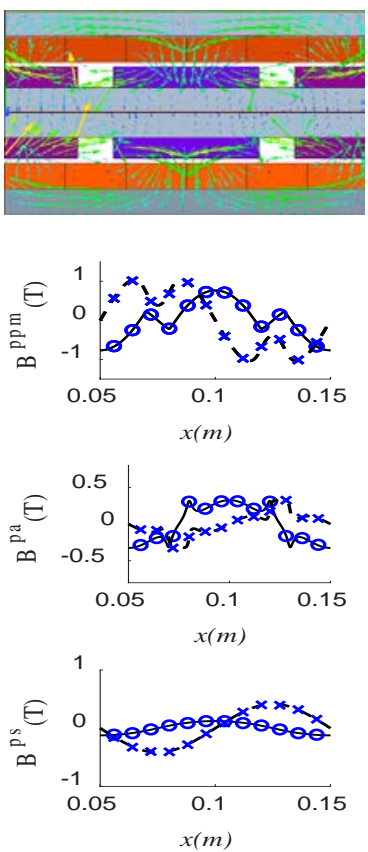

Bar magnets in shifting directions
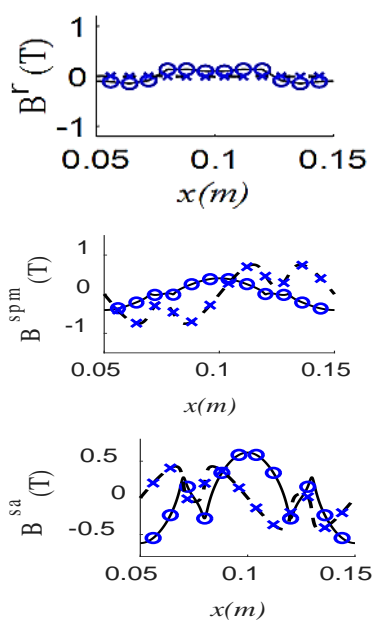

(b): Secondary sub-regions of the presented AFPMBM

o Numerical results of the normal component of flux density - - Analytical results of the tangential components of flux density $\times$ Numerical results of the tangential component of flux density - Analytical results of the normal component of flux density

Fig. 4. Analytical and numerical open circuit results of magnetic flux density distribution in motor under the study

\section{Conclusions}

An exact 2-D analytical sub-domain model for calculating the magnetic fields of a slotless AFPMBM is proposed in this paper based on the sub-domain method. The analytical procedure has been done in two separate steps including the effects of PMs and armature currents, respectively. Various magnetization patterns such as parallel, ideal Halbach, 2-segment Halbach and bar magnet in shifting direction have been considered to investigate 
the effects of PMs on the magnetic flux density distribution in each sub-region. The FEM is utilized to confirm the accuracy of the proposed analytical model and the extracted results reveal a suitable agreement between both analytical and numerical models. The benefits of the analytical models are described where less computational time of the analytical models was determined by employing a straightforward comparison of analytical and numerical simulation time and the analytical model was 11 times faster than the FEM.

\section{Appendix}

Imposing the boundary conditions between two adjacent sub-regions based on continuity of the tangential components of the magnetic field intensity and normal components of magnetic flux density are formed as follows:

a: Interface between primary exterior and primary stator at $y=y_{4}$

$$
\begin{aligned}
& \mu_{r}^{s} b_{n}^{p e} \sinh \left(\alpha_{n} y_{4}\right)-a_{n}^{p s} \cosh \left(\alpha_{n} y_{4}\right)-b_{n}^{p s} \sinh \left(\alpha_{n} y_{4}\right)=0 \\
& \mu_{r}^{s} d_{n}^{p e} \sinh \left(\alpha_{n} y_{4}\right)-c_{n}^{p s} \cosh \left(\alpha_{n} y_{4}\right)-d_{n}^{p s} \sinh \left(\alpha_{n} y_{4}\right)=0 \\
& b_{n}^{p e} \cosh \left(\alpha_{n} y_{4}\right)-a_{n}^{p s} \sinh \left(\alpha_{n} y_{4}\right)-b_{n}^{p s} \cosh \left(\alpha_{n} y_{4}\right)=0 \\
& d_{n}^{p e} \cosh \left(\alpha_{n} y_{4}\right)-c_{n}^{p s} \sinh \left(\alpha_{n} y_{4}\right)-d_{n}^{p s} \cosh \left(\alpha_{n} y_{4}\right)=0
\end{aligned}
$$

b: Interface between primary stator and primary winding at $y=y_{3}$

$$
\begin{aligned}
& \mu_{r}^{s} a_{n}^{p w} \cosh \left(\alpha_{n} y_{3}\right)+\mu_{r}^{s} b_{n}^{p w} \sinh \left(\alpha_{n} y_{3}\right)-a_{n}^{p s} \cosh \left(\alpha_{n} y_{3}\right)-b_{n}^{p s} \sinh \left(\alpha_{n} y_{3}\right)=0 \\
& \mu_{r}^{s} c_{n}^{p w} \cosh \left(\alpha_{n} y_{3}\right)+\mu_{r}^{s} d_{n}^{p w} \sinh \left(\alpha_{n} y_{3}\right)-c_{n}^{p s} \cosh \left(\alpha_{n} y_{3}\right)-d_{n}^{p s} \sinh \left(\alpha_{n} y_{3}\right)=0 \\
& a_{n}^{p w} \sinh \left(\alpha_{n} y_{3}\right)+b_{n}^{p w} \cosh \left(\alpha_{n} y_{3}\right)+\frac{\mu_{0}}{\alpha_{n}^{2}} J_{2 n}-a_{n}^{p s} \sinh \left(\alpha_{n} y_{3}\right)-b_{n}^{p s} \cosh \left(\alpha_{n} y_{3}\right)=0 \\
& c_{n}^{p w} \sinh \left(\alpha_{n} y_{3}\right)+d_{n}^{p w} \cosh \left(\alpha_{n} y_{3}\right)+\frac{\mu_{0}}{\alpha_{n}^{2}} J_{1 n}-c_{n}^{p s} \sinh \left(\alpha_{n} y_{3}\right)-d_{n}^{p s} \cosh \left(\alpha_{n} y_{3}\right)=0
\end{aligned}
$$

c: Interface between primary winding and primary air-gap at $y=y_{2}$

$$
\begin{aligned}
& a_{n}^{p w} \cosh \left(\alpha_{n} y_{2}\right)+b_{n}^{p w} \sinh \left(\alpha_{n} y_{2}\right)-a_{n}^{p a} \cosh \left(\alpha_{n} y_{2}\right)-b_{n}^{p a} \sinh \left(\alpha_{n} y_{2}\right)=0 \\
& c_{n}^{p w} \cosh \left(\alpha_{n} y_{2}\right)+d_{n}^{p w} \sinh \left(\alpha_{n} y_{2}\right)-c_{n}^{p a} \cosh \left(\alpha_{n} y_{2}\right)-d_{n}^{p a} \sinh \left(\alpha_{n} y_{2}\right)=0 \\
& a_{n}^{p w} \sinh \left(\alpha_{n} y_{2}\right)+b_{n}^{p w} \cosh \left(\alpha_{n} y_{2}\right)+\frac{\mu_{0}}{\alpha_{n}^{2}} J_{2 n}-a_{n}^{p a} \sinh \left(\alpha_{n} y_{2}\right)-b_{n}^{p a} \cosh \left(\alpha_{n} y_{2}\right)=0 \\
& c_{n}^{p w} \sinh \left(\alpha_{n} y_{2}\right)+d_{n}^{p w} \cosh \left(\alpha_{n} y_{2}\right)+\frac{\mu_{0}}{\alpha_{n}^{2}} J_{1 n}-c_{n}^{p a} \sinh \left(\alpha_{n} y_{2}\right)-d_{n}^{p a} \cosh \left(\alpha_{n} y_{2}\right)=0
\end{aligned}
$$

d: Interface between primary air-gap and primary PMs at $y=y_{1}$

$$
\begin{aligned}
& \mu_{r}^{P M} a_{n}^{p a} \cosh \left(\alpha_{n} y_{1}\right)+\mu_{r}^{P M} b_{n}^{p a} \sinh \left(\alpha_{n} y_{1}\right)-a_{n}^{p p m} \cosh \left(\alpha_{n} y_{1}\right)-b_{n}^{p p m} \sinh \left(\alpha_{n} y_{1}\right)=0 \\
& \mu_{r}^{P M} c_{n}^{p a} \cosh \left(\alpha_{n} y_{1}\right)+\mu_{r}^{P M} d_{n}^{p a} \sinh \left(\alpha_{n} y_{1}\right)-c_{n}^{p p m} \cosh \left(\alpha_{n} y_{1}\right)-d_{n}^{p p m} \sinh \left(\alpha_{n} y_{1}\right)=0 \\
& a_{n}^{p a} \sinh \left(\alpha_{n} y_{1}\right)+b_{n}^{p a} \cosh \left(\alpha_{n} y_{1}\right)-a_{n}^{p p m} \sinh \left(\alpha_{n} y_{1}\right)-b_{n}^{p p m} \cosh \left(\alpha_{n} y_{1}\right)-\frac{\mu_{0}}{\alpha_{n}} m_{x n}=0 \\
& c_{n}^{p a} \sinh \left(\alpha_{n} y_{1}\right)+d_{n}^{p a} \cosh \left(\alpha_{n} y_{1}\right)-c_{n}^{p p m} \sinh \left(\alpha_{n} y_{1}\right)-d_{n}^{p p m} \cosh \left(\alpha_{n} y_{1}\right)+\frac{\mu_{0}}{\alpha_{n}} m_{y n}=0
\end{aligned}
$$

e: Interface between primary $P M$ s and rotor at $y=y_{0}$

$$
\begin{aligned}
& \mu_{r}^{P M} a_{n}^{r} \cosh \left(\alpha_{n} y_{0}\right)+\mu_{r}^{P M} b_{n}^{r} \sinh \left(\alpha_{n} y_{0}\right)-\mu_{r}^{r} a_{n}^{p p m} \cosh \left(\alpha_{n} y_{0}\right)-\mu_{r}^{r} b_{n}^{p p m} \sinh \left(\alpha_{n} y_{0}\right)=0 \\
& \mu_{r}^{P M} c_{n}^{r} \cosh \left(\alpha_{n} y_{0}\right)+\mu_{r}^{P M} d_{n}^{r} \sinh \left(\alpha_{n} y_{0}\right)-\mu_{r}^{r} c_{n}^{p p m} \cosh \left(\alpha_{n} y_{0}\right)-\mu_{r}^{r} d_{n}^{p p m} \sinh \left(\alpha_{n} y_{0}\right)=0
\end{aligned}
$$




$$
\begin{aligned}
& a_{n}^{r} \sinh \left(\alpha_{n} y_{0}\right)+b_{n}^{r} \cosh \left(\alpha_{n} y_{0}\right)-a_{n}^{p p m} \sinh \left(\alpha_{n} y_{0}\right)-b_{n}^{p p m} \cosh \left(\alpha_{n} y_{1}\right)-\frac{\mu_{0}}{\alpha_{n}} m_{x n}=0 \\
& c_{n}^{r} \sinh \left(\alpha_{n} y_{0}\right)+d_{n}^{r} \cosh \left(\alpha_{n} y_{0}\right)-c_{n}^{p p m} \sinh \left(\alpha_{n} y_{0}\right)-d_{n}^{p p m} \cosh \left(\alpha_{n} y_{0}\right)+\frac{\mu_{0}}{\alpha_{n}} m_{y n}=0
\end{aligned}
$$

$f$ : Interface between rotor and secondary $P M s$ at $y=-y_{0}$

$$
\begin{aligned}
& \mu_{r}^{P M} a_{n}^{r} \cosh \left(-\alpha_{n} y_{0}\right)+\mu_{r}^{P M} b_{n}^{r} \sinh \left(-\alpha_{n} y_{0}\right)-\mu_{r}^{r} a_{n}^{s p m} \cosh \left(-\alpha_{n} y_{0}\right)-\mu_{r}^{r} b_{n}^{s p m} \sinh \left(-\alpha_{n} y_{0}\right)=0 \\
& \mu_{r}^{P M} c_{n}^{r} \cosh \left(-\alpha_{n} y_{0}\right)+\mu_{r}^{P M} d_{n}^{r} \sinh \left(-\alpha_{n} y_{0}\right)-\mu_{r}^{r} c_{n}^{s p m} \cosh \left(-\alpha_{n} y_{0}\right)-\mu_{r}^{r} d_{n}^{s p m} \sinh \left(-\alpha_{n} y_{0}\right)=0 \\
& a_{n}^{r} \sinh \left(-\alpha_{n} y_{0}\right)+b_{n}^{r} \cosh \left(-\alpha_{n} y_{0}\right)-a_{n}^{s p m} \sinh \left(-\alpha_{n} y_{0}\right)-b_{n}^{s p m} \cosh \left(-\alpha_{n} y_{1}\right)-\frac{\mu_{0}}{\alpha_{n}} m_{x n}=0 \\
& c_{n}^{r} \sinh \left(-\alpha_{n} y_{0}\right)+d_{n}^{r} \cosh \left(-\alpha_{n} y_{0}\right)-c_{n}^{s p m} \sinh \left(-\alpha_{n} y_{0}\right)-d_{n}^{s p m} \cosh \left(-\alpha_{n} y_{0}\right)+\frac{\mu_{0}}{\alpha_{n}} m_{y n}=0
\end{aligned}
$$

g: Interface between secondary PMs and secondary air-gap at $y=-y_{1}$

$$
\begin{aligned}
& \mu_{r}^{P M} a_{n}^{s a} \cosh \left(-\alpha_{n} y_{1}\right)+\mu_{r}^{P M} b_{n}^{s a} \sinh \left(-\alpha_{n} y_{1}\right)-a_{n}^{s p m} \cosh \left(-\alpha_{n} y_{1}\right)-b_{n}^{s p m} \sinh \left(-\alpha_{n} y_{1}\right)=0 \\
& \mu_{r}^{P M} c_{n}^{s a} \cosh \left(-\alpha_{n} y_{1}\right)+\mu_{r}^{P M} d_{n}^{s a} \sinh \left(-\alpha_{n} y_{1}\right)-c_{n}^{s p m} \cosh \left(-\alpha_{n} y_{1}\right)-d_{n}^{s p m} \sinh \left(-\alpha_{n} y_{1}\right)=0 \\
& a_{n}^{s a} \sinh \left(-\alpha_{n} y_{1}\right)+b_{n}^{s a} \cosh \left(-\alpha_{n} y_{1}\right)-a_{n}^{s p m} \sinh \left(-\alpha_{n} y_{1}\right)-b_{n}^{s p m} \cosh \left(-\alpha_{n} y_{1}\right)-\frac{\mu_{0}}{\alpha_{n}} m_{x n}=0 \\
& c_{n}^{s a} \sinh \left(-\alpha_{n} y_{1}\right)+d_{n}^{s a} \cosh \left(-\alpha_{n} y_{1}\right)-c_{n}^{s p m} \sinh \left(-\alpha_{n} y_{1}\right)-d_{n}^{s p m} \cosh \left(-\alpha_{n} y_{1}\right)+\frac{\mu_{0}}{\alpha_{n}} m_{y n}=0
\end{aligned}
$$

h: Interface between secondary air-gap and secondary winding at $y=-y_{2}$

$$
\begin{aligned}
& a_{n}^{s w} \cosh \left(-\alpha_{n} y_{2}\right)+b_{n}^{s w} \sinh \left(-\alpha_{n} y_{2}\right)-a_{n}^{s a} \cosh \left(-\alpha_{n} y_{2}\right)-b_{n}^{s a} \sinh \left(-\alpha_{n} y_{2}\right)=0 \\
& c_{n}^{s w} \cosh \left(-\alpha_{n} y_{2}\right)+d_{n}^{s w} \sinh \left(-\alpha_{n} y_{2}\right)-c_{n}^{s a} \cosh \left(-\alpha_{n} y_{2}\right)-d_{n}^{s a} \sinh \left(-\alpha_{n} y_{2}\right)=0 \\
& a_{n}^{s w} \sinh \left(-\alpha_{n} y_{2}\right)+b_{n}^{s w} \cosh \left(-\alpha_{n} y_{2}\right)+\frac{\mu_{0}}{\alpha_{n}^{2}} J_{2 n}-a_{n}^{s a} \sinh \left(-\alpha_{n} y_{2}\right)-b_{n}^{s a} \cosh \left(-\alpha_{n} y_{2}\right)=0 \\
& c_{n}^{s w} \sinh \left(-\alpha_{n} y_{2}\right)+d_{n}^{s w} \cosh \left(-\alpha_{n} y_{2}\right)+\frac{\mu_{0}}{\alpha_{n}^{2}} J_{1 n}-c_{n}^{s a} \sinh \left(-\alpha_{n} y_{2}\right)-d_{n}^{s a} \cosh \left(-\alpha_{n} y_{2}\right)=0
\end{aligned}
$$

$i$ : Interface between secondary winding and secondary stator at $y=-y_{3}$

$$
\begin{aligned}
& \mu_{r}^{s} a_{n}^{s w} \cosh \left(-\alpha_{n} y_{3}\right)+\mu_{r}^{s} b_{n}^{s w} \sinh \left(-\alpha_{n} y_{3}\right)-a_{n}^{s s} \cosh \left(-\alpha_{n} y_{3}\right)-b_{n}^{s s} \sinh \left(-\alpha_{n} y_{3}\right)=0 \\
& \mu_{r}^{s} c_{n}^{s w} \cosh \left(-\alpha_{n} y_{3}\right)+\mu_{r}^{s} d_{n}^{s w} \sinh \left(-\alpha_{n} y_{3}\right)-c_{n}^{s s} \cosh \left(-\alpha_{n} y_{3}\right)-d_{n}^{s s} \sinh \left(-\alpha_{n} y_{3}\right)=0 \\
& a_{n}^{s w} \sinh \left(-\alpha_{n} y_{3}\right)+b_{n}^{s w} \cosh \left(-\alpha_{n} y_{3}\right)+\frac{\mu_{0}}{\alpha_{n}^{2}} J_{2 n}-a_{n}^{s s} \sinh \left(-\alpha_{n} y_{3}\right)-b_{n}^{s s} \cosh \left(-\alpha_{n} y_{3}\right)=0 \\
& c_{n}^{s w} \sinh \left(-\alpha_{n} y_{3}\right)+d_{n}^{s w} \cosh \left(-\alpha_{n} y_{3}\right)+\frac{\mu_{0}}{\alpha_{n}^{2}} J_{1 n}-c_{n}^{s s} \sinh \left(-\alpha_{n} y_{3}\right)-d_{n}^{s s} \cosh \left(-\alpha_{n} y_{3}\right)=0
\end{aligned}
$$

$j$ : Interface between secondary stator and secondary exterior at $y=-y_{4}$

$$
\begin{aligned}
& \mu_{r}^{s} a_{n}^{s e} \cosh \left(-\alpha_{n} y_{4}\right)-a_{n}^{s s} \cosh \left(-\alpha_{n} y_{4}\right)-b_{n}^{s s} \sinh \left(-\alpha_{n} y_{4}\right)=0 \\
& \mu_{r}^{s s} c_{n}^{s e} \cosh \left(-\alpha_{n} y_{4}\right)-c_{n}^{s s} \cosh \left(-\alpha_{n} y_{4}\right)-d_{n}^{s s} \sinh \left(-\alpha_{n} y_{4}\right)=0 \\
& a_{n}^{s e} \sinh \left(-\alpha_{n} y_{4}\right)-a_{n}^{s s} \sinh \left(-\alpha_{n} y_{4}\right)-b_{n}^{s s} \cosh \left(-\alpha_{n} y_{4}\right)=0 \\
& c_{n}^{s e} \sinh \left(-\alpha_{n} y_{4}\right)-c_{n}^{s s} \sinh \left(-\alpha_{n} y_{4}\right)-d_{n}^{s s} \cosh \left(-\alpha_{n} y_{4}\right)=0
\end{aligned}
$$




\section{References}

[1] Mahmoudi A, Rahim NA, Hew WP. Axial-flux permanent-magnet machine modeling, design, simulation, and analysis. Scientific Research and Essays. 2011;6(12):2525-2549.

[2] Benlamine R, Dubas F, Randi SA, Lhotellier D, Espanet C. 3-D numerical hybrid method for PM eddycurrent losses calculation: Application to axial-flux PMSMs. IEEE Transactions on Magnetics. 2015;51(7):110.

[3] Zhang M, Cai B, Zhao X, Wang Z. Three-dimensional magnetic field analysis of micromotor by fast Fourier transform. IEEE Transactions on Magnetics. 1999;35(5):3685-3687.

[4] Thiagarajan V, Hsieh KT. Computation of magnetic fields using a 2-D hybrid finite-element/boundary element algorithm and comparison with analytical solutions. IEEE Transactions on Magnetics. 2005;41(1):393-397.

[5] Zhilichev YN. Calculation of 3D magnetic field of disk-type micromotors by integral transformation method. IEEE Transactions on Magnetics. 1996;32(1): 248-253.

[6] Ajily E, Abbaszadeh K, Ardebili M. Three-dimensional field reconstruction method for modeling axial flux permanent magnet machines. IEEE Transactions on Energy Conversion. 2015;30(1):199-207.

[7] Vahaj A, Rahideh A, Moayed-Jahromi H, Ghaffari A. Exact two-dimensional analytical calculations for magnetic field, electromagnetic torque, UMF, back-EMF, and inductance of outer rotor surface inset permanent magnet machines. Mathematical and Computational Applications. 2019;24(1):24.

[8] Chan TF, Xie S, Lai LL. Analysis of magnetic field in an axial-flux permanent-magnet synchronous generator using an analytical method. In: 2006 12th Biennial IEEE Conference on Electromagnetic Field Computation. IEEE. 2006.p. 146-146.

[9] Barakat G, Sambatra EJ, Azzouzi J, Dakyo B. Three dimensionnal analytical computation of magnetic field in single phase axial flux permanent magnet synchronous machine. In:2002 IEEE International Magnetics Conference (INTERMAG). IEEE. 2002.p. 500-500.

[10] Liu X, Hu H, Zhao J, Belahcen A, Tang L, Yang L. Analytical solution of the magnetic field and EMF calculation in ironless BLDC motor. IEEE Transactions on Magnetics. 2016;52(2):1-10.

[11] Sung SY, Jeong JH, Park YS, Choi JY, Jang SM. Improved analytical modeling of axial flux machine with a double-sided permanent magnet rotor and slotless stator based on an analytical method. IEEE Transactions on Magnetics. 2012;48(11):2945-2948.

[12] Choi JY, Park YS, Jang SM. Magnetic field computation of axial flux permanent magnet machines with Halbach and axially magnetized rotor using quasi-3-D analysis modeling. In: Digests of the 2010 14th Biennial IEEE Conference on Electromagnetic Field Computation. IEEE. 2010.p. 1-1.

[13] Furlani EP, Knewtson MA. A three-dimensional field solution for permanent-magnet axial-field motors. IEEE Transactions on Magnetics. 1997;33(3):2322-2325.

[14] Azzouzi J, Barakat G, Dakyo B. Quasi-3-D analytical modeling of the magnetic field of an axial flux permanent-magnet synchronous machine. IEEE Transactions on Energy Conversion. 2005;20(4):746-752.

[15] Azzouzi J, Barakat G, Dakyo B. Analytical modeling of an axial flux permanent magnet synchronous generator for wind energy application. In: IEEE International Conference on Electric Machines and Drives, 2005. IEEE. 2005.p. 1255-1260.

[16] Azzouzi J, Barakat G, Dakyo B. Quasi-3-D analytical modeling of the magnetic field of an axial flux permanent-magnet synchronous machine. IEEE Transactions on Energy Conversion. 2005;20(4):746-752.

[17] Chan TF, Xie S, Lai LL. Computation of no-load and armature reaction fields of an axial-flux permanentmagnet synchronous generator. In:2007 IEEE International Electric Machines \& Drives Conference. IEEE. 2007.Vol. 1, p. 1-6.

[18] Virtic P, Pisek P, Marcic T, Hadziselimovic M, Stumberger B. Analytical analysis of magnetic field and back electromotive force calculation of an axial-flux permanent magnet synchronous generator with coreless stator. IEEE Transactions on Magnetics. 2008;44(11):4333-4336.

[19] Karim NA, Azzouzi J, Barakat G, Dakyo B. Complete analytical modeling of an axial flux PM synchronous machine for wind energy application. In: 2006 12th International Power Electronics and Motion Control Conference. IEEE. 2006.p. 996-1001.

[20] Chan TF, Lai LL. Computation of air-gap field in an axial-flux permanent-magnet machine using the method of images. In:2009 IEEE International Electric Machines and Drives Conference. IEEE. 2009. p. 1647-1651.

[21] Bellara A, Amara Y, Barakat G, Reghem P. Analytical modelling of the magnetic field in axial flux permanent magnet machines with semi-closed slots at no load. In: The XIX International Conference on Electrical Machines-ICEM 2010. IEEE. 2010.p. 1-6.

[22] Choi JY, Park YS, Ko KJ, Jang SM. Electromagnetic analysis of double-sided axial flux permanent magnet motor with ring-wound type slotless stator based on analytical modeling. In: 2010 International Conference on Electrical Machines and Systems. IEEE. 2010.p. 1213-1217. 
[23] Choi JY, Lee SH, Ko KJ, Jang SM. Improved analytical model for electromagnetic analysis of axial flux machines with double-sided permanent magnet rotor and coreless stator windings. IEEE Transactions on Magnetics. 2011;47(10):2760-2763.

[24] Tiegna H, Bellara A, Amara Y, Barakat G. Analytical modeling of the open-circuit magnetic field in axial flux permanent-magnet machines with semi-closed slots. IEEE Transactions on Magnetics. 2012;48(3):12121226.

[25] Huang Y, Ge B, Dong J, Lin H, Zhu J, Guo Y. 3-D analytical modeling of no-load magnetic field of ironless axial flux permanent magnet machine. IEEE Transactions on Magnetics. 2012;48(11):2929-2932.

[26] Bellara A, Tiegna H, Amara Y, Barakat G. On load analytical modelling of the magnetic field for axial flux surface-inset permanent magnet machines with semi-closed slots. In: 2012 XXth International Conference on Electrical Machines. IEEE. 2012.p. 2852-2858.

[27] Hong SA, Choi JY, Jang SM, Jung KH. Torque analysis and experimental testing of axial flux permanent magnet couplings using analytical field calculations based on two polar coordinate systems. IEEE Transactions on Magnetics. 2014;50(11):1-4

[28] Park HJ, Woo DK, Jung SY, Jung HK. Modeling and analysis of magnet skew in axial flux permanent-magnet motor via field reconstruction method. In: 2016 19th International Conference on Electrical Machines and Systems (ICEMS). IEEE. 2016.p. 1-4.

[29] Parviainen A, Niemela M, Pyrhonen J. Modeling of axial flux permanent-magnet machines. IEEE Transactions on Industry Applications. 2004;40(5):1333-1340.

[30] Ajily E, Abbaszadeh K, Ardebili M. Three-dimensional field reconstruction method for modeling axial flux permanent magnet machines. IEEE Transactions on Energy Conversion. 2015;30(1):199-207.

[31] Khoobroo A, Fahimi B, Pekarek SD. A new field reconstruction method for permanent magnet synchronous machines. In: 2008 34th Annual Conference of IEEE Industrial Electronics. IEEE. 2008.p. 2009-2013.

[32] Jin P, Yuan Y, Minyi J, Shuhua F, Heyun L, Yang H, Ho SL. 3-D analytical magnetic field analysis of axial flux permanent-magnet machine. IEEE Transactions on Magnetics. 2014;50(11):1-4.

[33] Ghaffari A, Rahideh A, Moayed-Jahromi H, Vahaj AA, Mahmoudi A, Soong WL. 2-D analytical model for outer-rotor consequent-pole brushless PM machines. IEEE Transactions on Energy Conversion. 2019;34(4):2226-2234.

[34] Zhu ZQ, Wu LJ, Xia ZP. An accurate subdomain model for magnetic field computation in slotted surfacemounted permanent-magnet machines. IEEE Transactions on Magnetics. 2010;46(4):1100-1115.

[35] Rahideh A, Ghaffari A, Barzegar A, Mahmoudi A. Analytical model of slotless brushless PM linear motors considering different magnetization patterns. IEEE Transactions on Energy Conversion. 2018;33(4):17971804.

[36] D'Angelo J, Chari MVK, Campbell P. Three-dimensional finite element solution for a permanent magnet axial-field machine. IEEE Transactions on Power Apparatus and Systems. 1983(1): 83-90.

[37] Alipour A, Moallem M. Analytical magnetic field analysis of axial flux permanent-magnet machines using Schwarz-Christoffel transformation. In: 2013 International Electric Machines \& Drives Conference. IEEE. 2013.p. 670-677.

[38] Nasar SA, Xiong G. Determination of the field of a permanent-magnet disk machine using the concept of magnetic charge. IEEE Transactions on Magnetics. 1988;24(3):2038-2044.

[39] Campbell P, Chari M, Angelo JD. Three-dimensional finite element solution of permanent magnet machines. IEEE Transactions on Magnetics. 1981;17(6):2997-2999.

[40] Tiegna H, Amara Y, Barakat G. A new quasi-3-D analytical model of axial flux permanent magnet machines. IEEE Transactions on Magnetics. 2014;50(2):817-820.

[41] Aydin M, Huang S, Lipo TA. Optimum design and 3D finite element analysis of nonslotted and slotted internal rotor type axial flux PM disc machines. In: 2001 Power Engineering Society Summer Meeting. IEEE. 2001.p. 1409-1416.

[42] Pop AA, Radulescu MM, Balan H, Chirca M, Zaharia V. Electromagnetic design and finite-element analysis of an axial-flux permanent-magnet machine. In: 2014 International Conference on Applied and Theoretical Electricity (ICATE). IEEE. 2014.p. 1-4.

[43] Dhifli M, Bali H, Laoubi Y, Barakat G, Amara Y. Analytical modeling of the magnetic field in axial field flux-switching permanent magnet machines at no-load. In: 2013 15th European Conference on Power Electronics and Applications (EPE). IEEE. 2013.p. 1-10.

[44] Furlani EP. Computing the field in permanent-magnet axial-field motors. IEEE Transactions on Magnetics. 1994;30(5):3660-3663.

[45] Bumby JR, Martin R, Mueller MA, Spooner E, Brown NL, Chalmers BJ. Electromagnetic design of axialflux permanent magnet machines. IEE Proceedings-Electric Power Applications. 2004,151(2):151-160.

[46] Chan TF, Lai LL, Xie S. Field computation for an axial flux permanent-magnet synchronous generator. IEEE Transactions on Energy Conversion. 2009;24(1):1-11. 
[47] Koechli C, Perriard Y. Analytical model for slotless permanent magnet axial flux motors. In: 2013 International Electric Machines \& Drives Conference. IEEE. 2013.p. 788-792.

[48] Chan TF, Wang W, Lai LL. Performance of an axial-flux permanent magnet synchronous generator from 3D finite-element analysis. IEEE Transactions on Energy Conversion. 2010;25(3):669-676.

[49] Qudsia J, Junaid I, Kwon BI. Analytical analysis of the magnetic field and no-load voltage for the double sided axial flux permanent magnet synchronous generator. In: Digests of the 2010 14th Biennial IEEE Conference on Electromagnetic Field Computation. IEEE. 2010.p. 1-1.

[50]Boroujeni ST, Mohammadi AA, Oraee A, Oraee H. Approach for analytical modelling of axial-flux PM machines. IET Electric Power Applications. 2016;10(6):441-450.

[51] Virtic P, Pisek P, Hadziselimovic M, Marcic T, Stumberger B. Torque analysis of an axial flux permanent magnet synchronous machine by using analytical magnetic field calculation. IEEE Transactions on Magnetics. 2009;45(3):1036-1039.

[52] Rahideh A, Korakianitis T. Analytical magnetic field distribution of slotless brushless PM motors. Part 2: Open-circuit field and torque calculations. IET Electric Power Applications. 2012;6(9):639-651.

[53] Rahideh A, Korakianitis T. Analytical calculation of open-circuit magnetic field distribution of slotless brushless PM machines. International Journal of Electrical Power \& Energy Systems. 2013;44(1):99-114.

[54] Ghaffari A. 2-D Analytical model for predicting magnetic flux distribution in slotless single-sided axial flux permanent-magnet synchronous machines. Journal of Modeling and Optimization. 2019;11(2):97-105.

[55] Shin KH, Cho HW, Lee SH, Choi JY. Armature reaction field and inductance calculations for a permanent magnet linear synchronous machine based on subdomain model. IEEE Transactions on Magnetics. 2017;53(6):1-4

(c) 2020 by the author(s). This work is licensed under a Creative Commons Attribution 4.0 International License (http://creativecommons.org/licenses/by/4.0/). Authors retain copyright of their work, with first publication rights granted to Tech Reviews Ltd. 